\title{
INCORPORAÇÃO DE RESÍDUO DE MASSA CERÂMICA CRUA EM CERÂMICA VERMELHA*
}

Alessandra Savazzini dos Reis ${ }^{1}$ Francisco Rolando Valenzuela-Diaz ${ }^{2}$

\section{Resumo}

A crescente preocupação em relação às áreas de proteção ambiental e ao alto consumo de argila, matéria-prima da cerâmica vermelha, ocasiona a indisponibilização progressiva de reservas de argilas no Brasil. Com isso, vê-se a necessidade de incorporar resíduos na massa argilosa para confecção de produtos de cerâmica vermelha. A indústria de cerâmica vermelha brasileira consome cerca de 10 milhões de toneladas de argila ao mês para produção de telhas e blocos. $\mathrm{Na}$ própria olaria são gerados resíduos, tal como, o resíduo de massa cerâmica crua originado na etapa de conformação das peças. Este trabalho visa incorporar o resíduo de massa cerâmica crua na massa argilosa usada em cerâmica vermelha. $O$ resíduo foi caracterizado por meio de ensaios de FRX, DRX, limites de Atterberg e distribuição de tamanho de partículas. Corpos de prova feitos com massa argilosa e resíduo em teores variando de $10 \%$ a $90 \%$ (massa) foram conformados, secos à $110^{\circ} \mathrm{C}$, queimados à $900^{\circ} \mathrm{C}$ em forno industrial e submetidos a ensaios de absorção de água, porosidade aparente, massa específica aparente e resistência à compressão. Os resultados evidenciaram que as propriedades cerâmicas avaliadas nos corpos de prova da massa argilosa incorporada com o resíduo atendem aos valores das argilas-padrão brasileiras para cerâmica vermelha.

Palavras-chave: Resíduo de massa cerâmica crua; Cerâmica vermelha; Incorporação; Resíduo.

\section{RAW CERAMIC MASS WASTE INCORPORATION IN STRUCTURAL CERAMIC Abstract}

The growing concern in relation to areas of environmental protection and high consumption of clay as raw-material of structural ceramic, causes progressive unavailability of clay reserves in Brazil. It confirms the need to incorporate waste in the clayey mass for making structural ceramic products. The Brazilian structural ceramic industry consumes about 10 million tons of clay per month for production of roof tiles and blocks. Wastes, such as the raw ceramic mass waste originated in the pieces conformation stage, are generated in the pottery itself. This paper aims to incorporate the raw ceramic mass waste in the clayey mass used in structural ceramic. The waste was tested by XRF, XRD, Atterberg limits and particle size distribution. Specimens with mixtures of clayey mass and waste of the contents ranging from $10 \%$ to $90 \%$ (weight) were conformed, dried at $110^{\circ} \mathrm{C}$ and burned at $900^{\circ} \mathrm{C}$ in the industrial furnace and, after that, tested for water absorption, apparent porosity, apparent specific mass and compressive strength. The results show that the ceramic properties evaluated in specimens of clayey mass incorporated with waste meet the requirements of the Brazilian standard clays for structural ceramics.

Keywords: Raw ceramic mass waste; Structural ceramic; Incorporation; Waste.

1 Engenheira Civil, Doutora, professora, Coordenadoria de Arquitetura e Urbanismo, IFES, Colatina, ES, Brasil.

2 Engenheiro Químico, Doutor, professor, PMT-EPUSP, São Paulo, SP, Brasil. 


\section{INTRODUÇÃO}

A crescente preocupação em relação ao meio ambiente, em especial às áreas de proteção ambiental, e ao alto consumo de argila, como matéria-prima da indústria de cerâmica vermelha, ocasiona a indisponibilização progressiva de reservas de argilas, embora exista grande volume de reservas no Brasil. Aliados a isso, há um conjunto de fatores que se apropriam de amplos espaços geográficos e competem com a mineração, tais como: a expansão urbana, o adensamento da ocupação agrícola e a demanda cada vez maior por recursos hídricos para abastecimento, irrigação e geração de energia [1]. A extração de argila para suprir as olarias é feita em jazidas de pequeno porte a céu aberto com escala de produção variando de 1.000 a 20.000 toneladas por mês com baixo valor de matéria-prima e alto custo de transporte [2].

A argila é um material natural, terroso, de granulometria fina, que geralmente adquire plasticidade quando umedecido com água. $E$ em proporções corretas de água e argila forma-se uma massa plástica suscetível à modelagem [3] para fabricação de produtos cerâmicos. Em função da formação e fatores de alteração durante consolidação, existe uma grande variedade de argilas com toda gama de cor, plasticidade e composição química [4], fatores esses que permitem a inserção de resíduos de diferentes processos industriais na mistura argilosa usada na cerâmica vermelha.

Com isso, vê-se a necessidade de incorporar resíduos na massa argilosa para confecção de produtos de cerâmica vermelha que consumam menos matéria-prima argilosa.

Devido às propriedades e abundância das argilas, não existem materiais naturais substitutos às argilas nos processos cerâmicos industriais; no entanto a tendência tecnológica verificada nos últimos anos é o uso de resíduo [2]; o que também contribui para diminuição do volume de resíduo disposto em aterros [5].

Sabe-se que a indústria de cerâmica vermelha brasileira consome cerca de 10 milhões de toneladas de argila ao mês para produção de 4 bilhões de blocos e 1,3 bilhões de telhas [6] que atendem ao setor da construção civil.

Na própria olaria são gerados resíduos, tal como, o resíduo de massa cerâmica crua originado na etapa de conformação das peças. O resíduo (Figura 1) é composto por rebarbas da prensagem de telhas e por blocos e telhas recém-conformados descartados por apresentarem defeitos visuais.

O resíduo em questão tem como principais constituintes, massa argilosa e resíduo de corte de granito usados como matérias-primas na produção de telhas e blocos cerâmicos. Estima-se que o resíduo de massa cerâmica crua representa cerca de $40 \%$ do volume da massa argilosa conformada [7].

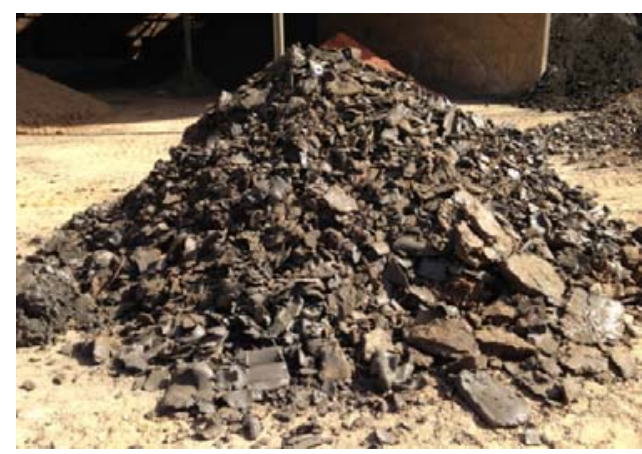

Figura 1. Pilha de resíduo de massa cerâmica crua 
Na pesquisa bibliográfica realizada, não houve registro de trabalhos científicos sobre a incorporação do resíduo de massa cerâmica crua nas próprias olarias ou em outras aplicações. Diversos trabalhos [8, 9, 10,11] existem sobre a utilização do chamote, denominação dada à massa argilosa queimada. Nesse sentido, o trabalho visa incorporar o resíduo de massa cerâmica crua na massa argilosa usada em cerâmica vermelha.

\section{MATERIAIS E MÉTODOS}

Amostras de resíduo de massa cerâmica crua e da massa argilosa foram coletadas em pilha de armazenagem de matérias-primas em fábrica de cerâmica vermelha situada no estado do Espírito Santo.

As amostras foram secas ao ar livre, destorroadas, homogeneizadas e quarteadas. Parte do material foi destinada para realização de ensaios de caracterização, e parte foi utilizada para confecção dos corpos de prova.

A caracterização do resíduo de massa cerâmica crua foi feita por meio de: fluorescência de raios $X(F R X)$ em espectrômetro marca Philips modelo PW2400; difração de raios $X(D R X)$ com fonte de cobre (radiação $K \alpha$ ) em aparelho Philips XPERT MPD; limites de Atterberg segundo normas da ABNT [12, 13]; e análise da distribuição de tamanho de partículas por difração a laser em equipamento Mastersizer 2000 da Malvern.

A identificação das amostras da DRX foi feita por comparação com arquivos do padrão Crystallographic Open Database (COD).

Com o resíduo (Figura 2 a) e a massa argilosa passados na peneira $n^{\circ} 40$ da ABNT (abertura de $0,42 \mathrm{~mm}$ ) foram feitas misturas da massa argilosa contendo resíduo em teores variando de $0 \%$ a $90 \%$ (massa); e conformados manualmente corpos de prova esféricos (Figura $2 \mathrm{~b}$ ) com massa seca de $10 \mathrm{~g}$, diâmetro médio de $2 \mathrm{~cm}$ e água suficiente para conformação, seguindo método desenvolvido no Laboratório de materiais não metálicos Professor Persio de Souza Santos (LPSS) do PMT/EPUSP.

O uso da metodologia de conformação de esferas para análise das propriedades cerâmicas em misturas argilosas se dá em função de ser uma metodologia simples e de baixo custo que pode ser desenvolvida na própria olaria [14].

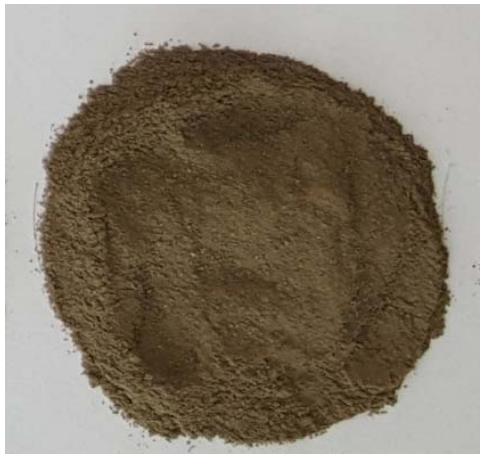

Figura 2 a. Resíduo de massa cerâmica crua passado na peneira ABNT ${ }^{\circ} 40$

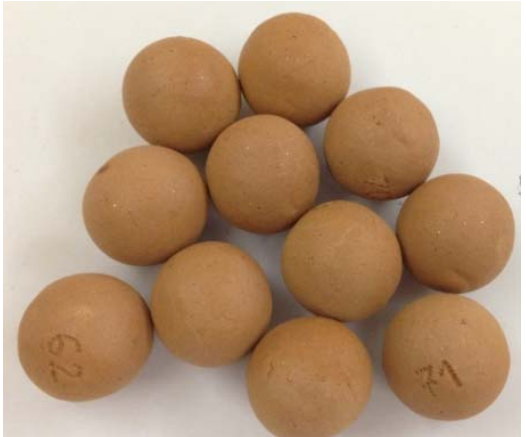

Figura 2 b. Corpos de prova esféricos queimados

Os corpos de prova esféricos foram secos em estufa à $110^{\circ} \mathrm{C}$ por $24 \mathrm{~h}$, sendo posteriormente verificada a perda de massa. Em seguida, parte dos corpos de prova foi queimada em forno industrial contínuo tipo túnel por $24 \mathrm{~h}$ a uma temperatura máxima na zona de queima de $900^{\circ} \mathrm{C}$ em fábrica de cerâmica vermelha situada no ES. 
A utilização do forno industrial teve como objetivo estabelecer condições ambientais e técnicas de fabricação para os corpos de prova da pesquisa semelhantes às utilizadas para os produtos cerâmicos.

Os corpos de prova queimados foram submetidos a ensaios de absorção de água, porosidade aparente, massa específica aparente, perda de massa, retração de queima de acordo com metodologia de Souza Santos [15], além da resistência à compressão medida em prensa Marcon com capacidade de $15 \mathrm{t}$.

\section{RESULTADOS E DISCUSSÃO}

\subsection{Caracterização do resíduo de massa cerâmica crua}

O resultado da análise química do resíduo de massa cerâmica crua (Tabela 1) é apresentado em termos de percentual em peso dos óxidos constituintes e da perda ao fogo.

Analisando os dados da Tabela pode-se verificar que o resíduo de massa cerâmica crua apresenta uma composição química com predominância de sílica e alumina, com valores $44,70 \%$ e $27,57 \%$, respectivamente. Neste caso, pode-se classificar o resíduo como um material sílico-aluminoso. Esses valores se situam dentro do intervalo estabelecido por Souza Santos [15] para matéria-prima de cerâmica vermelha. O teor de Ferro de 8,96\% confere a cor avermelhada após a queima. $O$ valor da perda ao fogo de $12,85 \%$ está situado no intervalo de $6,00 \%$ a $15,70 \%$ [15]. Verifica-se também a presença dos óxidos alcalinos $\mathrm{K}_{2} \mathrm{O}$ e $\mathrm{Na}_{2} \mathrm{O}$ em função do resíduo de massa cerâmica crua conter em sua composição o resíduo do corte de granito.

Tabela 1. Composição química por FRX do resíduo de massa cerâmica crua

\begin{tabular}{cc}
\hline \multicolumn{2}{c}{ Composição em óxidos (\%) } \\
\hline $\mathrm{SiO}_{2}$ & 44,70 \\
\hline $\mathrm{Al}_{2} \mathrm{O}_{3}$ & 27,57 \\
\hline $\mathrm{Fe}_{2} \mathrm{O}_{3}$ & 8,96 \\
\hline $\mathrm{CaO}$ & 1,01 \\
\hline $\mathrm{K}_{2} \mathrm{O}$ & 1,56 \\
\hline $\mathrm{MgO}$ & 0,72 \\
\hline $\mathrm{MnO}$ & 0,06 \\
\hline $\mathrm{Na}_{2} \mathrm{O}$ & 0,65 \\
\hline $\mathrm{P}_{2} \mathrm{O}_{5}$ & 0,16 \\
\hline $\mathrm{TiO}_{2}$ & 1,22 \\
\hline $\mathrm{BaO}$ & 0,26 \\
\hline $\mathrm{ZnO}$ & 0,22 \\
\hline $\mathrm{Co}_{2} \mathrm{O}_{3}$ & $<0,1$ \\
\hline $\mathrm{Cr}_{2} \mathrm{O}_{3}$ & $<0,1$ \\
\hline $\mathrm{PbO}_{2}$ & $<0,1$ \\
\hline $\mathrm{SrO}$ & $<0,1$ \\
\hline $\mathrm{ZrO}+\mathrm{HfO}_{2}$ & $<0,1$ \\
\hline $\mathrm{Perda}$ ao fogo & 12,85 \\
\hline
\end{tabular}

No difratograma de raios-X do resíduo (Figura 3) verificou-se a presença de quartzo, forma cristalina do $\mathrm{SiO}_{2}$ encontrado na análise química. Foram detectados também picos de mica, feldspato e ortoclasio. Sabendo-se que quartzo, feldspato e mica figuram como principais constituintes das rochas graníticas [16] pode-se confirmar que o resíduo em questão contém o resíduo de corte de granito em sua composição. Foram encontrados picos de caulinita e goetita presentes na massa argilosa que 
origina o resíduo. Com isso, o resultado do DRX corrobora o resultado encontrado na FRX e a informação de que o resíduo de massa cerâmica crua possui resíduo de corte de granito em sua composição.

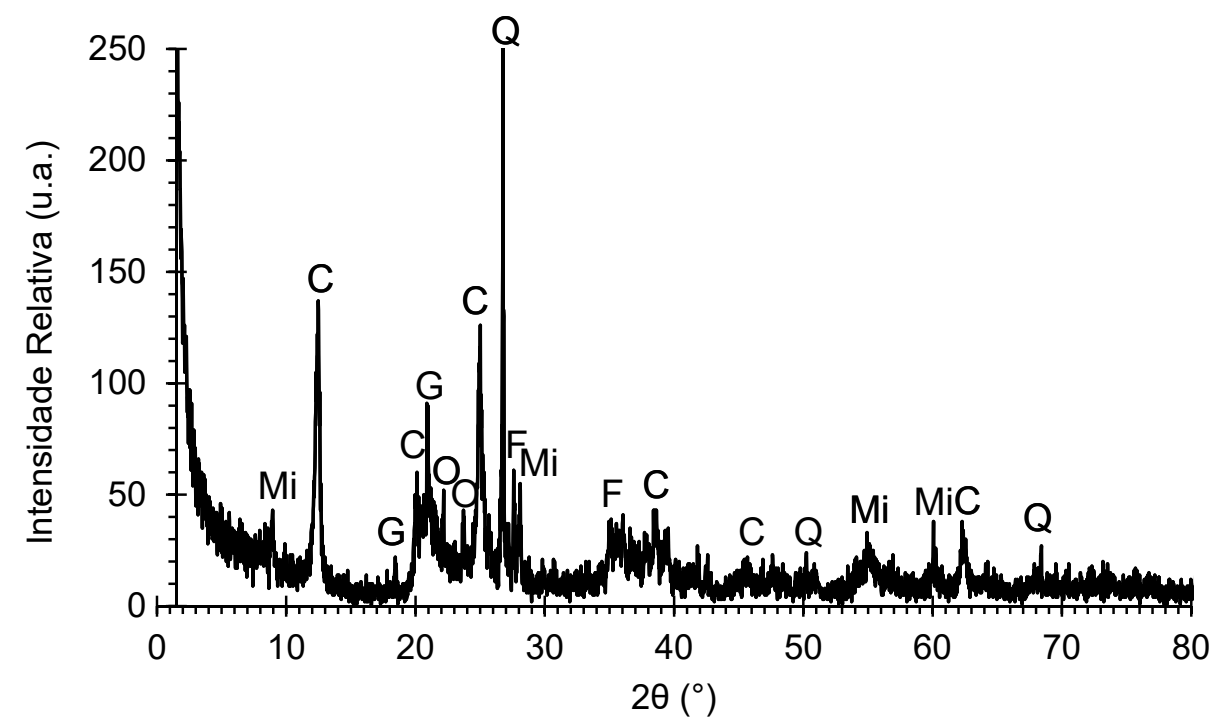

Figura 3. DRX do resíduo de massa cerâmica crua

Nota: C - caulinita (COD 1011045); F - feldspato (COD 9001898); G - goetita (COD 1008768); Mi mica (COD 9000819); O - ortoclásio (COD 9006347); Q - quartzo (COD 9012602)

Os limites de Atterberg são apresentados na Tabela 2. O índice de plasticidade (IP) obtido de $20,75 \%$ indica que o resíduo de massa cerâmica crua tem alta plasticidade (IP maior que 15), valor adequado para materiais cerâmicos moldados por extrusão [17]. O alto valor de IP pode demandar maior quantidade de água para extrusão e demandar maior tempo de secagem das peças.

O limite de plasticidade de $29,45 \%$ se situou entre $9 \%$ e $56 \%$ prescrito por Souza Santos [15] para argilas cauliníticas, concordando com o resultado de DRX, que indicou picos de caulinita.

Tabela 2. Limites de Atterberg

\begin{tabular}{cccc}
\hline Características & $\begin{array}{c}\text { Limite de } \\
\text { liquidez (\%) }\end{array}$ & $\begin{array}{c}\text { Limite de } \\
\text { plasticidade (\%) }\end{array}$ & $\begin{array}{c}\text { Índice de } \\
\text { plasticidade IP(\%) }\end{array}$ \\
\hline Resíduo & 50,20 & 29,45 & 20,75 \\
\hline
\end{tabular}

A distribuição de tamanho de partícula do resíduo de massa cerâmica crua está apresentada na Figura 4. O gráfico do resíduo se apresenta com 10\% dos grãos menores que 2,10 $\mu \mathrm{m}$ (diâmetro efetivo). Pode-se obter através dos dados da curva de distribuição de tamanho de partícula que $11 \%$ dos grãos são menores que $2 \mu \mathrm{m}$; $64 \%$ dos grãos tem tamanhos entre $2 \mu \mathrm{m}$ e $20 \mu \mathrm{m}$ e $25 \%$ dos grãos são maiores que $20 \mu \mathrm{m}$. Verifica-se com isso, que o resíduo possui baixa "fração argila" (grãos menores que $2 \mu \mathrm{m}$ ) e que a maior parte dos grãos se situa no intervalo de tamanho de partículas associadas ao "silte" (grãos entre $2 \mu \mathrm{m}$ e $20 \mu \mathrm{m}$ ). Nessa faixa de tamanho de grãos pode haver mineral argiloso que proporciona plasticidade [18].

Como a plasticidade é influenciada pela fina granulometria e pela composição química do material argiloso, pode-se dizer que a alta plasticidade apresentada pelo resíduo se deve provavelmente à presença de matéria orgânica, pois a fração argila encontrada foi baixa. 


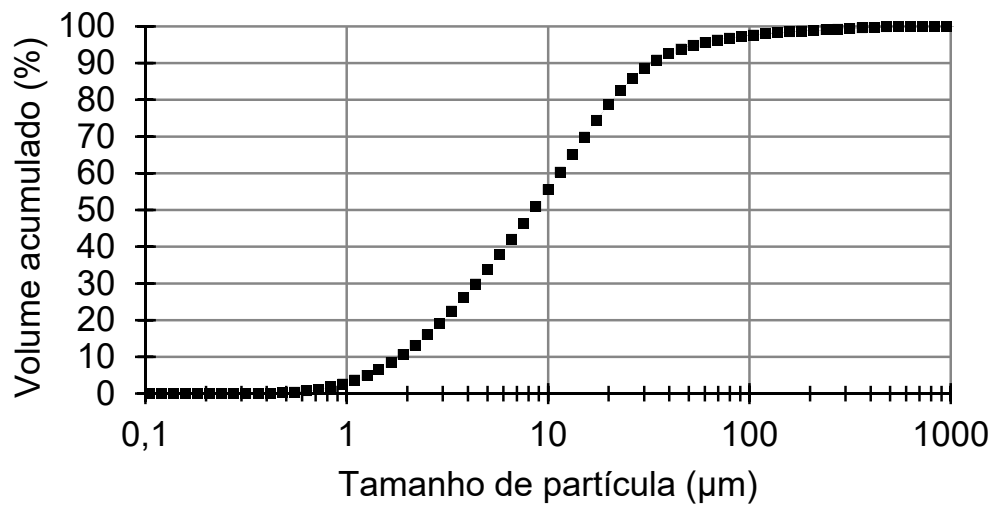

Figura 4. Gráfico de frequência da análise de tamanho de partícula do resíduo de massa cerâmica crua

\subsection{Propriedades cerâmicas dos corpos de prova esféricos}

Os resultados obtidos nos corpos de prova esféricos apresentados na Tabela 3 mostram que: perda de secagem (PS), perda ao fogo (PF), retração volumétrica de queima $\left(R V_{Q}\right)$, absorção de água $(A A)$ e porosidade aparente (PA) diminuíram seus valores com o aumento do teor de resíduo, enquanto os valores da massa específica aparente (MEA) aumentaram, o que confirma o aumento da densificação nos corpos de prova promovido pelo resíduo de massa cerâmica crua, que por sua vez possui resíduo de corte de granito na composição.

Pode ser verificado que o resíduo promoveu uma maior estabilidade dimensional nos corpos de prova durante a queima em função da diminuição nos valores apresentados de retração e perda ao fogo, fato relatado também em outras pesquisas $[19,20]$.

Os valores da resistência à compressão nos corpos de prova após a queima $(\sigma)$ aumentaram com a incorporação do resíduo sendo máxima no teor de $70 \%$ (massa). Considerando os valores de referência utilizados [15] para corpos de prova prismáticos, todos os valores encontrados de absorção de água e massa específica aparente atendem aos valores das argilas-padrão brasileiras após queima, cujos valores são: $25 \%$ e 1,5 a $2,0 \mathrm{~g} / \mathrm{cm}^{3}$, respectivamente [15]. A porosidade aparente atende ao limite máximo de $35 \%$ a partir do teor de resíduo de $60 \%$ (massa) [15].

Tabela 3. Resultados médios das propriedades cerâmicas nas esferas

\begin{tabular}{cccccccc}
\hline Resíduo(\%) & $\mathbf{P S}(\%)$ & $\mathbf{P F}(\%)$ & $\mathbf{A A}(\%)$ & $\mathbf{P A}(\%)$ & $\mathbf{M E A}\left(\mathbf{g} / \mathbf{c m}^{3}\right)$ & $\mathbf{R V} \mathbf{Q}(\%)$ & $\boldsymbol{\sigma}(\mathbf{M P a})$ \\
\hline 0 & 25,69 & 12,23 & 24,78 & 37,69 & 1,51 & 6,20 & 2,99 \\
\hline 10 & 25,00 & 12,68 & 24,30 & 37,11 & 1,51 & 6,19 & 3,15 \\
\hline 20 & 24,79 & 12,62 & 23,96 & 36,92 & 1,54 & 5,75 & 3,25 \\
\hline 30 & 24,62 & 12,35 & 23,69 & 36,22 & 1,54 & 6,57 & 3,22 \\
\hline 40 & 24,62 & 12,20 & 23,15 & 36,39 & 1,57 & 6,26 & 3,41 \\
\hline 50 & 24,52 & 12,19 & 22,56 & 35,43 & 1,57 & 6,19 & 3,81 \\
\hline 60 & 24,48 & 12,10 & 22,25 & 34,85 & 1,57 & 5,71 & 3,84 \\
\hline 70 & 24,10 & 12,05 & 21,69 & 34,29 & 1,58 & 5,64 & 4,29 \\
\hline 80 & 22,57 & 11,89 & 21,64 & 24,42 & 1,59 & 5,61 & 3,70 \\
\hline 90 & 22,61 & 11,76 & 21,10 & 33,59 & 1,59 & 5,50 & 3,92 \\
\hline Referência* $^{*}$ & - & 6.00 a 15.70 & $\leq 25.00$ & $\leq 35.00$ & 1.50 a 2.00 & 2 a 17 & - \\
\hline
\end{tabular}

Nota: $P S$ = perda de secagem; $P F=$ perda ao fogo; $A A=$ absorção de água; $P A$ = porosidade aparente; $M E A=$ massa especifica aparente; $R V_{Q}=$ retração volumétrica de queima; $\sigma$ = resistência à compressão.

*Nota: Referência Souza Santos [15]. 
A caracterização química e mineralógica do resíduo de massa cerâmica crua demonstrou que o argilomineral predominante é a caulinita, o resíduo pode ser considerado sílico-aluminoso e contém teores de quartzo e de ferro adequados para fabricação de cerâmica vermelha.

$\mathrm{O}$ alto teor de sílica presente no resíduo promoveu a estabilidade dimensional nos corpos de prova confirmada com os baixos valores encontrados de retração de queima e perda ao fogo.

A amostra possui distribuição de tamanho de partícula com baixo teor da fração argila, mas apresenta plasticidade adequada para uso em cerâmica vermelha.

Os valores encontrados de absorção de água, massa específica aparente e porosidade aparente atendem aos valores das argilas-padrão brasileiras para uso em cerâmica vermelha.

Os resultados do estudo evidenciam que o resíduo de massa cerâmica crua pode ser incorporado à massa argilosa na fabricação de produtos de cerâmica vermelha, pois a incorporação do resíduo causou melhorias nas propriedades analisadas em relação à massa argilosa original (sem conter resíduo).

Com isso, a incorporação do resíduo de massa cerâmica crua na massa argilosa é uma alternativa sustentável ambientalmente para a indústria de cerâmica vermelha.

\section{REFERÊNCIAS}

1 Ministério das Minas e Energia (MME). Relatório técnico 32 - Perfil da argila. 2009. [acesso em 14 abr. 2016] 30p. Disponível em: http://www.mme.gov.br/documents/ 1138775/1256650/P23_RT32_Perfil_da_Argila.pdf/b6fc71dc-3c0a-4eb1-b2a5f62b2c3bec0.

2 Ministério do Desenvolvimento, Indústria e Comércio Exterior do BR (MDIC) Cerâmica vermelha - Termo de referência. [Acesso em 14 abr. 2016]. Disponível em: http://www.mdic.gov.br/arquivos/dwnl_1295436730.pdf.

3 Callister, WD Ciência e engenharia de materiais: uma introdução. $5^{a}$ edição. Rio de Janeiro: LTC, 2002. 589p.

4 Petrucci EGR. Materiais de construção. $4^{\text {a }}$ Edição. Porto Alegre: Editora Globo; 1979. 435p.

5 Reis, AS, Della-Sagrillo, VP,Valenzuela-Diaz, FR. Green structural ceramic with addition of raw clay waste. In: TMS145th Annual Meeting \& Exhibition. Nashville Tennessee - EUA. 2016. 6p.

6 Associação Nacional da Indústria Cerâmica (ANICER). Dados oficiais. RJ. 2016. [Acesso em 12 abr. 2016]. Disponível em: http://www.anicer.com.br/index.asp?pg= institucional.asp\&secao=3\&categoria $=60 \&$ selMenu $=4$.

7 Reis, AS, Della-Sagrillo, VP,Valenzuela-Diaz, FR. Caracterização de resíduo de massa cerâmica crua para aproveitamento em cerâmica vermelha. In: $70^{\circ}$ Congresso Anual da ABM. Rio de Janeiro - BR. 2016. 8p.

8 Vieira AAP. Estudo do aproveitamento de resíduos de cerâmica vermelha como substituição pozolânica em argamassas e concretos. 2005. 107f. Dissertação (Engenharia Urbana) - UFPB. 2005.

9 Gouveia FP, Sposto RM. Incorporação de chamote em massa cerâmica para a produção de blocos. Um estudo das propriedades físico-mecânicas. Revista Cerâmica. 2009; 55: 415-419.

10 Redivo IM. Utilização de resíduo de cerâmica vermelha em misturas com solo para construção de camadas de pavimentos com baixo volume de tráfego. 2011. $160 f$.

Dissertação (Engenharia Civil) - UFSC. 2011. 
11 Fastofski DC, Schafer M, Teles RD, Kulakowski MP, Gonzales MAS, Kazmierczak CS. Metodologia de caracterização de resíduo de cerâmica vermelha para emprego como material pozolânico em pasta de cimento. [acesso em 12 mar. 2015]. In: $5^{\circ}$ Fórum Mundial de Resíduos Sólidos. São Leopoldo. RS. 2014. Disponível em: http://www.5firs.institutoventuri.org.br/arquivo/download?ID_ARQUIVO=66

12 Associação Brasileira de Normas Técnicas (ABNT). NBR 6459: Solo - determinação de limite de liquidez. Rio de Janeiro, 1984.

13 . NBR 7180: Solo - determinação do limite de plasticidade. Rio de Janeiro, 1984.

14 Miyahara, RY; Furlan, GHRH; Valenzuela-Diaz, FR; Toffoli, SM. Correlação entre medidas de resistência mecânica de corpos de prova de argila conformados manualmente e por prensagem uniaxial. In: $48^{\circ}$ Congresso Brasileiro de Cerâmica, 2004, Curitiba. Anais... Curitiba: ABCERAM, 2004.

15 Souza Santos P. Tecnologia das argilas. Vol.1-2. São Paulo: Edgard Blucher, 1975.

16 Manhães, JPVT, Moreira, JMS, Holanda, JNF. Variação microestrutural de cerâmica vermelha incorporada com resíduo de rocha ornamental. Revista Cerâmica, São Paulo, v. 55, p. 371-378, 2009.

17 Caputo, HP. Mecânica dos solos e suas aplicações. 6ª edição. v1. Rio de Janeiro: LTC, 2011. 234p

18 Vieira, CMF; Sales, HF; Monteiro, SN. Effect of illitic flux clay addition in red ceramic of kaolinitic clays. Revista Cerâmica, São Paulo, n. 50, p. 239-246, 2004.

19 Taguchi SP, Santos JC, Gomes TM, Cunha NA. Avaliação das propriedades tecnológicas de cerâmica vermelha incorporada com resíduo de rocha ornamental proveniente do tear de fio diamantado. Revista Cerâmica. 2014; 60: 291-296.

20 Reis, AS. Produção de telha cerâmica com incorporação simultânea de resíduo de beneficiamento de granito, lama de alto forno e resíduo de massa cerâmica crua. 2015. 123p. Tese (Engenharia Metalúrgica e de Materiais) - PMT/EPUSP. 2015. 